Fernando Verdugo, s.j.

Profesor

Universidad Alberto Hurtado

\title{
Fe y modernidad en América Latina: La teología de Juan Luis Segundo
}

En el contexto de la preparación para la V Conferencia General del Episcopado Latinoamericano y del Caribe, puede ser importante recoger el aporte de aquellos teólogos que han contribuido, con su pensamiento, a acortar la brecha o "ruptura entre Evangelio y cultura" en la región (1). El Documento de Participación, como parte del proceso de preparación a dicha Conferencia, nos invita a evocar a los "discípulos y misioneros santos", hombres y mujeres, jóvenes y adultos que han vivido de manera ejemplar el ideal cristiano en el continente (175-192). Además de su testimonio, dada la complejidad de los cambios culturales que estamos experimentando -cambios que el mismo Documento destaca claramente (94-104)-, sería conveniente también recoger el legado de quienes han puesto su inteligencia al servicio de la fe. Para hacerlo, ya no hay que acudir allende las fronteras del continente: los tenemos en casa. En este artículo quisiera traer a la memoria a un teólogo uruguayo, el jesuita Juan Luis Segundo (1925-1996), de cuya "pascua" acabamos de celebrar diez años. Su pensamiento teológico se hizo sentir, directa e indirectamente, en las anteriores Conferencias. Al recordar su legado teológico, tal vez pueda ser también un aporte en la V Conferencia en Aparecida, Brasil, en mayo de 2007.

En términos generales, habría que decir que desde un comienzo la reflexión metódica y crítica sobre la fe que desarrolló Juan Luis Segundo estuvo siempre orientada a enfrentar asuntos propios del continente latinoamericano, sin que por ello desconociera que tales asuntos estaban afectados por otros de carácter mundial, a los que cada vez más prestó mayor atención. Fue sin duda cofundador y claro exponente de la teología de la liberación, a pesar de las reservas que presentó en cierto momento hacia determinadas corrientes. Segundo planteó su quehacer teológico como una ayuda para que los cristianos reflexionaran sobre su fe comprometida con las necesidades y desafíos que les planteaba el contexto. Más que en la academia, su reflexión teológica la realizó en el seno de algunas comunidades laicales, sobre todo de clase media urbana.

(1) "La ruptura entre Evangelio y cultura" era, para el papa Pablo VI, "el drama de nuestro tiempo". De ahí que en 1975 invitara a los cristianos a "hacer todos los esfuerzos con vistas a una generosa evangelización de la cultura, o más exactamente de las culturas" (Evangelii Nuntiandi, 18-20). 
No se puede, en pocas páginas, dar cuenta del rico y prolífico legado teológico de Juan Luis Segundo (2). Me limitaré a dar a conocer cómo en su tiempo y lugar buscó articular más estrechamente el Evangelio y las culturas; en particular, la fe y la cultura moderna que impactaba a amplios sectores de Montevideo y de otras urbes latinoamericanas. Tempranamente Juan Luis Segundo fue consciente del "drama" del que hablaría Pablo VI en 1975. En efecto, desde comienzos de los años sesenta nuestro teólogo apelaba a un cambio en las "estructuras o esquemas mentales" en América Latina, sobre todo a nivel de las masas, porque a su juicio dificultaban, por ejemplo, el establecimiento del vínculo entre la eficacia del servicio histórico a los hombres y la eficacia del orden de la salvación (3). Dicho de otro modo, los esfuerzos por dignificar la vida humana no se asociaban con el Evangelio de Jesús, debido a unos "esquemas mentales" o a la cultura imperante. Pero Segundo no solo urgió a liberar la teología y la pastoral de paradigmas obsoletos e, incluso, contraproducentes; puso, además, el Evangelio en nuevas coordenadas culturales.

En lo que sigue, entonces, expondré las principales coordenadas o mediaciones culturales que conforman la matriz desde donde Juan Luis Segundo piensa y significa la salvación cristiana en América Latina. Dicha matriz surge de un análisis semiótico, cuya perspectiva y método no cabe desarrollar aquí. Bastaría decir que, tratándose la cultura de sistemas de significación históricamente construidos y transmitidos, a través de los cuales se comunican y desarrollan unos conocimientos y se orienta la acción al interior de un determinado colectivo humano, la mejor manera de acceder a lo cultural serían los métodos capaces de sacar a luz dichos sistemas. Las mediaciones culturales desentrañadas serán presentadas en forma de paradigmas o relaciones de oposición, de modo que se destaquen las alternativas en juego. Veamos, pues, algunos paradigmas de pensamiento que condicionan la elaboración teológica de Juan Luis Segundo (4).

\section{UNIVERSO EN EVOLUCIÓN VS. UNIVERSO ESTÁTICO}

El paradigma que subyace en su forma de concebir el espacio o ámbito en el que se desarrolla su discurso teológico, es el de un "universo en evolución vs. universo estático". Por ejemplo, en El hombre de hoy ante Jesús de Nazaret, la obra de mayor envergadura de Segundo aparecida en los ochenta, podemos constatar que la evolución del universo no solo es un tema del que se habla, sino también un dato tenido por verdadero y utilizado en la reflexión teológica. Para Segundo, por ejemplo, algunos de los "planteamientos radicalmente nuevos" desde los cuales hay que plantearse la significación de Jesús de Nazaret para el hombre de hoy,

(2) Una biografía y bibliografía pueden encontrarse en: http://www.juanluissegundo.com

(3) Véanse, por ejemplo, algunos artículos de la década de los sesenta recopilados en De la sociedad a la teología, Ed. Carlos Lohlé, Buenos Aires 1970.

(4) Los objetivos, método y resultados del estudio brevemente esbozado aquí, puede verse en: Fernando Verdugo, Relectura de la Salvación cristiana en Juan Luis Segundo. Estudio de las mediaciones culturales subyacentes a su soteriología de los años ochenta, Universidad Pontificia Comillas, Madrid 1996; publicada también en Colección: Anales de la Facultad de Teología LIV, Pontificia Universidad Católica de Chile, Santiago 2003. 
serían: el asumir el desafío, del cual el hombre es realmente consciente solo a partir los últimos cincuenta años, de dirigir la evolución universal y biológica, al menos en la reducida escala de nuestro planeta; el desempeñar esa nueva tarea de manera ecológica y política; y el aceptar la lentitud de los complejos procesos evolutivos (5). Sin duda, pues, para Segundo la evolución del universo es un dato "objetivo" con el que hay que contar, y con el que de hecho cuenta, en la reflexión teológica.

Es difícil no caer en la cuenta del influjo de ciertas teorías científicas en esta percepción dinámico-evolutiva del universo. Con mayor razón si el mismo Segundo reconoce y asume ese influjo. Se trata de teorías de la evolución biológica y universal que, de acuerdo con Segundo, existían a nivel de hipótesis desde hace más de un siglo y que solo en los últimos cincuenta años se han convertido en tesis (6). Sin embargo, confiesa él mismo, "no existe aún un verdadero contexto evolucionista, con categorías acuñadas por la cultura y usadas como 'lo obvio' en el plano de la cultura humana" (7). Los planteamientos de científicos del campo de la biología, como J. P. Lamark, Ch. Darwin, A. Wallace, J. Monod, Gregory Bateson, etc., son acogidos (y discutidos) con frecuencia en las obras de Segundo. También hay ecos de las teorías de científicos del campo de la astrofísica, tales como S. Hawking y Trinh Xuan Thuan, sobre todo en la década de los noventa (8). Además, cabe mencionar a P. Teilhard de Chardin, quien ya había intentado anteriormente reconciliar la concepción evolutiva del universo con el mensaje cristiano, y cuyo pensamiento recoge Segundo y le sirve de plataforma para el suyo propio (9).

Por último, señalemos que tal concepción del universo atraviesa o estructura no solo la forma de pensar la salvación, sino también otros temas relacionados con la fe cristiana -por ejemplo, los dogmas de la Iglesia (10)-, dado que la misma epistemología de Segundo se ha hecho evolutiva.

\section{HISTORIA SIGNIFICATIVA $V S$. HISTORIA EVENEMENCIAL}

Estrechamente vinculada con la concepción evolutiva del universo está la principal forma de pensar el tiempo subyacente en su discurso teológico. El tiempo se entiende fundamentalmente como "historia". En cuanto tal, no se refiere solo al pasado ni a un mero repertorio de acontecimientos. Decir "historia", como forma de concebir el tiempo, implica un sentido: ya sea porque la historia encierra unos significados a descubrir, ya sea porque está orientada hacia un futuro hacia el cual se avanza. Esta concepción del tiempo como historia con sentido es lo que nos ha

(5) Cf. J. L. Segundo, El hombre de hoy ante Jesús de Nazaret, tomo II/2, Ed. Cristiandad, Madrid 1982, pp. 803-815.

(6) Cf. ib., pp. 804-805.

(7) Ib., pp. 961-962.

(8) El mayor diálogo de Segundo con astrofísicos puede percibirse en obras como: ¿Qué mundo? ¿Qué hombre? ¿Qué Dios?, Sal Terrae, Santander 1993.

(9) Véase, por ejemplo, en El hombre de hoy ante Jesús de Nazaret, tomo II/2, el uso abundante que hace Segundo del pensamiento de Teilhard de Chardin, aunque también tome distancia con respecto a él (sobre todo pp. 821-957).

(10) Cf. J. L. Segundo, El dogma que libera, Sal Terrae, Santander 1989. 
llevado a expresar este paradigma de pensamiento en términos de "historia significativa $v s$. historia evenemencial" (11).

Puede ser ilustrativo el siguiente mensaje extraído de una obra de J. L. Segundo, donde explicita cómo ha de entenderse la "historia":

"la historia 'significativa' (...) no es lo que burlonamente definíamos en la escuela a espaldas del maestro: la sucesión de sucesos sucedidos sucesivamente (...) Para inyectar sentido en ese caos, para convertir la sucesión de sucesos en 'historia', hay que puntuarla. Y para ello depende uno en parte del texto que está ahí, y en parte también de uno mismo" (12).

"Puntuar" es organizar los diversos sucesos y descubrirles o concederles un sentido. La historia, para ser tal, debe contemplar los hechos más el significado que a estos les "inyecta" el ser humano. En definitiva, el tiempo que se considera es el que lleva anejo un significado, en función del futuro. A ese tiempo se le denomina "historia", y no al mero inventario de sucesos o eventos del pasado.

Este tiempo se concibe también como "historia significativa" porque a esta se la presenta ligada de manera indisociable a lo trascendente. Lo trascendente no se ha de buscar al margen, fuera o después de la historia, sino en ella. De este modo, es una la historia del encuentro de Dios con el hombre y la historia que avanza, junto al universo, hacia una mayor plenitud en el futuro.

Además, la reflexión y propuesta de salvación cristiana en la obra de Segundo está mediada por una concepción de un tiempo que avanza, puesto que lo mejor siempre se ubica en el futuro y no en el pasado. Y avanzaría en espiral, si tomamos en cuenta que a veces -se hace notar- hay ciertos acontecimientos y acciones que implican retrocesos. La "historia", pues, la principal forma de concebir el tiempo, al igual que el universo, avanza hacia su plenitud junto con (y en gran parte gracias a) la humanidad. Si bien esta concepción del tiempo como historia única y en progreso está en la tradición judeocristiana (v. g., Dios como creador, la historia como lugar de encuentro con Dios, etc.), es también heredera de los planteamientos al respecto de la Modernidad. Constituye un paradigma que aflora y no parece cuestionarse en el corpus de obras de J. L. Segundo (13).

(11) "Historia evenemencial" designa la opción de ciertos historiadores de considerar los hechos o eventos (de ahí "evenemencial") de manera puntual y no dentro de procesos de larga duración en que los hechos cobrarían sentido.

(12) El Dogma que libera, ob. cit., p. 68.

(13) Esta forma de concebir el tiempo y la historia no es extraña en el concierto de la teología occidental. Andrés Tornos constata que gran parte de los teólogos contemporáneos que abordan el tema de la relación entre esperanzas históricas y esperanza cristiana (Bultmann, Rahner, Pannemberg, Moltmann, Gutiérrez, etc.) no parecen "poner en duda el convencimiento de la cultura moderna ilustrada, según la cual la humanidad avanza en la historia, de tal forma que puede discernirse el valor de lo que ocurre por la forma como todo ello se integra en ese avance" (Escatología I, Universidad Pontificia Comillas, Madrid 1989, pp. 132-133). 


\section{ANTROPOCENTRISMO VS. TEOCENTRISMO}

Otra importante mediación cultural que emerge de la lectura semiótica de la obra de Segundo sería la mentalidad que sitúa al hombre como punto de partida de todo lo que se reflexiona y comunica: es decir, son cuestiones relativas a la existencia histórica del ser humano las que están en el centro de este discurso teológico. De ahí que denominemos "antropocentrismo" a la forma de esta mediación. En cambio, el elemento alternativo del paradigma, i. e. "teocentrismo", expresaría un tipo de razonamiento que sitúa a Dios en el centro de todo desarrollo teológico. Un ejemplo de este último tipo de razonamiento sería la teología tomista, que parte deductivamente de unas ideas acerca de Dios tenidas por verdaderas.

Este antropocentrismo, detectado en la obra de Segundo, parece incidir en que la salvación aparezca, antes que nada, como algo que atañe al hombre y su existencia histórica. A un conocedor del pensamiento teológico le parecerá evidente que afirmemos que este énfasis en lo humano es una manifestación del "giro antropológico" que se ha producido desde hace algún tiempo en el conjunto del discurso teológico occidental, a la zaga en parte del mismo giro que ha tenido lugar en otros campos del saber humano, de la filosofía en particular.

\section{SERES HUMANOS VS. SOBRENATURALES; CRISTIANOS Y NO CRIS- TIANOS VS. SOLO CRISTIANOS; NO POBRES VS. POBRES}

El análisis cultural pone de relieve los actores que importan en los distintos "escenarios" o representaciones culturales del mundo. Pues bien, en primer lugar hay que destacar que en los distintos "escenarios" demarcados temporalmente como historia, las acciones que inciden en la trama son casi exclusivamente acciones atribuidas a seres humanos. Estamos, pues, lejos de una representación mítica del mundo; es decir, de un mundo en que seres sobrenaturales intervendrían para incidir en su curso. Más aún, Dios no aparece interviniendo directamente en el mundo ni en la historia. Su manifestación está siempre mediada por acciones o personajes humanos. Nos situamos, así, ante una visión moderna del mundo en la que, desde Newton en adelante, se percibe como movido por leyes naturales y no por intervenciones sobrenaturales.

En segundo lugar, habría que destacar en la obra de Segundo un intento por determinar y consolidar la identidad del protagonista o "sujeto operador" (en lenguaje semiótico) de las transformaciones históricas valoradas positivamente. En la representación que realiza Segundo del escenario de América Latina, y también del universal, los cristianos no son los únicos "protagonistas". Todo ser humano puede colaborar creativamente en la obra creadora y liberadora iniciada por Dios. Más aún, en este discurso teológico hay cristianos que incluso figuran en el papel de "oponentes".

Este ejercicio de "negociación de identidad" -corriente en tiempos de cambio cultural-, ocupa un lugar muy importante en la obra de Segundo y, ciertamente, en otros teólogos contemporáneos (14). Digamos de paso que, en su modo de negociar

(14) Piénsese, por ejemplo, en los “cristianos anónimos” de Karl Rahner. 
la identidad, la distinción que hace Segundo entre "fe antropológica" y "fe religiosa" es clave (15). Ella le servirá de fundamento para concebir que tanto "cristianos" como "no cristianos" estén en posición de protagonista. Tanto cristianos como no cristianos pueden estar adoptando el mismo valor absoluto para orientar sus vidas, pueden tener una misma "fe antropológica". La diferencia radicaría en que los primeros relacionan el valor absoluto con Dios, y los segundos no. En efecto, para Segundo, uno de los elementos específicos de la "fe religiosa" sería el convencimiento de que el valor absoluto de una determinada escala de valores adoptada por la fe "antropológica" tiene relación con Dios y su revelación.

En tercer lugar, en el corpus teológico analizado aflora otra negociación de identidad. Esta tendría lugar a comienzo de los años ochenta dentro de ese colectivo representado por los teólogos de la liberación, donde se pretendía responder a la siguiente pregunta: ¿Son o no los pobres sujetos operadores de la salvación/liberación histórica (16)? En la obra de Segundo, los protagonistas de la salvación se distinguen de aquellos que figuran como "destinatarios" de la misma; es decir, de la mayoría del pueblo pobre, los pobres y oprimidos de América Latina. En general, a los destinatarios se les atribuyen funciones de carácter pasivo: por ejemplo, "ser organizados nuevamente en una sociedad distinta y solidaria". Quienes figuran como protagonistas o continuadores de la misión salvífica de Jesús en favor de los pobres, no pertenecen a este último grupo.

A modo de conclusión de este apartado: partiendo de la consideración de seres humanos como sujetos operadores de las acciones de transformación, pasando por su delimitación como cristianos y no cristianos, y terminando en su especificación como no pobres, digamos que, en la medida en que refleja planteamientos cada vez más particulares o de grupos más restringidos, se trata de elementos cada vez menos culturales; es decir, menos socialmente compartidos. Ya comentaremos esto al final.

\section{PRIORIDAD A LA LIBERTAD VS. PRIORIDAD A LA LEY}

La libertad ocupa un lugar importante en el discurso teológico de Segundo: figura como un don otorgado por Dios (el "destinador", en lenguaje semiótico) a todo ser humano. Asumirla y ejercerla sin temor emerge como condición o requisito (una "competencia", en lenguaje semiótico) para que los hombres que encarnan el papel de protagonista puedan cumplir las tareas encomendadas (la "performancia"): liberar o salvar a los pobres y oprimidos, crear proyectos de amor que lleven el universo inacabado hacia su término.

El que se conceda tal importancia a la libertad es ciertamente un factor cultural, un fenómeno ampliamente compartido. La teología, en cuanto discurso social específico, también ha sido receptiva de esa centralidad que le otorga la Modernidad

(15) Véase, por ejemplo, El hombre de hoy ante Jesús de Nazaret, tomo I (Ed. Cristiandad, Madrid 1982) y, mejor, La historia perdida y recuperada de Jesús de Nazaret, tres primeros capítulos (Sal Terrae, Santander 1991).

(16) Esta "negociación de identidad" se encuentra desarrollada, por ejemplo, en Teología abierta, tomo III, sobre todo en el Cap. V: "Dos teologías de la liberación”, y en el VII: "El pueblo, sujeto de su historia" (Ed. Cristiandad, Madrid 1984). 
a la libertad en Occidente, constituyéndola igualmente en uno de los valores más apreciados y objeto de enorme atención en su propio discurso. Sin embargo, el corpus teológico de Segundo no es un mero eco de ese interés por la libertad. Encontramos en él un intento por reajustar o "negociar" el significado que la libertad debiera tener, precisamente en función de una determinada concepción de la salvación.

La negociación del sentido de la libertad se verifica, por ejemplo, en Teología Abierta, tomo III (17). Allí cuestiona que, tanto en el ámbito protestante como católico, primen unas determinadas 'teologías de la gracia' que, en lugar de favorecer la "función creadora" de la libertad, generan miedo y parálisis en las personas por lo que pueden hacer con ella. En estas teologías, a su juicio, la libertad se entiende básicamente como requisito para superar una prueba impuesta por Dios, y no como requisito para actuar creativamente y colaborar así en la salvación que incluye al cosmos y a la humanidad entera. Dicho esto, es importante señalar que no estaríamos aquí ante un paradigma cultural ampliamente compartido. Más bien estamos ante el intento de introducir un nuevo paradigma o modo de concebir la libertad. Hasta qué punto el modo de entender la libertad desarrollado por Segundo está presente en nuestra cultura, es algo que habría que analizar.

El paradigma que hemos puesto de relieve en el título se debe a que en un mundo que se percibe en proceso de evolución, la libertad se valora por encima de la ley. La ley, más que expresión de un orden permanente en un mundo que se tiene por acabado, figura en el discurso de Segundo como una ayuda para el ejercicio de la libertad. La ley es fruto de la experiencia acumulada, que orienta el devenir de la humanidad y del universo entero en camino hacia una mayor plenitud.

\section{MALES HiSTÓRICOS VS. NATURALES; ESTRUCTURALES VS. PUN- TUALES}

Como bien dice Edward Schillebeeckx: "las ideas y expectativas de salvación de un pueblo están marcadas por las experiencias negativas de la humanidad" (18); y, además, que cualquier "experiencia es siempre experiencia interpretada" (19). Una de las tareas del análisis cultural consiste, entonces, en desentrañar cómo se interpretan esas experiencias negativas.

Lo primero que habría que destacar es que en la obra de Segundo priman los males del ámbito socioeconómico: "miseria y opresión" de las grandes mayorías de América Latina. Las causas de estos males no serían naturales: es decir, el ser pobre u oprimido no es una fatalidad con que la naturaleza marca a unos seres humanos, ni menos algo querido por Dios. Son más bien males que tienen causas históricas: es decir, fruto de la interacción humana en la historia. Además, el discurso intenta mostrar el carácter estructural ( $v s$. puntual o circunstancial) de lo que produce los males relevados: son unas "estructuras externas e internas" las que los generan. Al

(17) Ib., véase, sobre todo, las pp. 219-275.

(18) E. SChillebeECKX, Jesús, la historia de un viviente, Ed. Cristiandad, Madrid ${ }^{2} 1983$, p. 15.

(19) E. SChillebeECKX, Cristo y los cristianos, Ed. Cristiandad, Madrid 1982, p. 23. 
analizar semióticamente este discurso teológico, constatamos que la situación socioeconómica se valora negativamente no tanto desde la mentalidad marxista -por cierto presente y asumida críticamente en el discurso de Segundo-, sino más bien desde otra mentalidad -“el cristianismo"- que, paradójicamente, ha convivido con tal situación. En efecto, es desde lo que el autor llama "el cristianismo" desde donde la situación se cualifica como "de miseria y opresión del hombre", "de injusticia" y sobre todo "de pecado" que no se debiera "tolerar". De alguna manera, pues, la forma como se perciben los males está medida con un código de raíz cristiana.

Ahora bien, siendo "el pecado" un código o producto cultural de raíz cristiana, aparece también en el discurso teológico de Segundo con otras particularidades. De nuevo estaríamos ante lo que en análisis cultural se llama una "negociación de significado": es decir, se busca ajustar el significado de pecado con la mentalidad evolutiva y moderna. En efecto, el pecado se lo describe como unas "fuerzas" que amenazan al hombre en su tarea de colaborar con Dios en la obra liberadora, en su función creadora de cara al universo incompleto. Esas fuerzas son el "temor a la libertad" que impide al hombre desplegar su creatividad; "las propias pasiones" del hombre, capaces de doblegarlo hasta convertirlo en su esclavo; y, sobre todo, los "instrumentos ya creados" que, con sus mecanismos, pueden desviar al hombre de su tarea histórica. El pecado, mediante tales fuerzas, conduce los proyectos del hombre a otro desenlace: a una "muerte en la historia", a un visible fracaso. Como se puede apreciar, el pecado no se percibe aquí como una "falta" del hombre en la supuesta prueba que Dios le impone en un universo ya acabado, sino más bien como un elemento constitutivo del proceso de evolución universal, con el que el hombre debe contar (y vencer) en su tarea de colaborar con Dios en su obra creadora.

Por último, hay que poner otro mal de relieve, pero subordinado a los anteriores, un mal muy característico de los textos de la década de los ochenta. Se trata de la "desesperanza" que afecta a quienes intentan transformar la situación de pobreza y opresión del continente latinoamericano; desesperanza que se manifiesta en frustración por políticas de desarrollo ineficaces, en acción violenta (subversiva) y en la "pasividad".

Así, pues, la experiencia e interpretación de los males en la obra teológica de Segundo están mediadas culturalmente por elementos de significación aportados por el marxismo, por la mentalidad evolutiva y, ciertamente, por el cristianismo presente en América Latina desde hace casi cinco siglos.

\section{UTOPÍA DE LA HUMANIZACIÓN VS. UTOPÍA DE LA RESTAURACIÓN}

El siguiente paradigma -“utopía de la humanización vs. utopía de la restauración"- pone de manifiesto la forma de entender el objetivo de la salvación y, al mismo tiempo, el valor que comporta dicho objetivo. En efecto, en la obra de Segundo "humanizar al hombre" y otras expresiones emparentadas ("humanización", "más humano/a"), además de ser algunas de las figuras con que se representa el objetivo de la acción transformadora o estado final que se quiere alcanzar, son términos mediante los cuales se expresa el valor de dicho estado o nueva situación. Por ejemplo, la misión o proyecto de Jesús es el Reino de Dios, cuya llegada implica 
sacar los pobres de una situación inhumana y hacerlos pasar a otra más humana. La "humanización" no es un valor más; es el valor que gobierna o aglutina a los demás.

El que "humanización" y sus términos afines constituye un dato cultural radica en el hecho de que aparece como un elemento de significación del discurso que se tematiza frecuentemente, pero que no se determina. Ni en el texto analizado ni en los textos sondeados se deja oír un cuestionamiento o una defensa de la "humanización del hombre" en cuanto objetivo y valor a conseguir. Al leer los diversos textos del corpus, da la impresión de que el autor pero también los posibles lectores entienden lo que significan los términos "humanización", "humanizar" y "más humano/a". Se trata de un "objeto-valor", cuyo "valor" (valga la redundancia) no se cuestiona y al que hay que tender.

Esta mediación cultural también está relacionada con la idea que desde la Modernidad en adelante se va teniendo acerca del hombre y de sus capacidades de transformación del mundo y su entorno; idea que poco a poco se iría extendiendo y haciéndose de "sentido común". Además, para darse una formulación del objetivo de la salvación en términos de una humanización, es necesario un contexto donde el hombre y sus posibilidades en la historia se entiendan de manera evolutiva o progresiva. Es necesario que el hombre deje de considerarse como especie fija, como "ser completo", y pase a entenderse como "ser en evolución". Si se lo considerara como especie fija, íntegra desde un comienzo, la salvación apuntaría a restaurar la integridad previamente dada y luego perdida. De ahí que, a nuestro juicio, la alternativa en juego sería "utopía de la restauración".

Las mediaciones culturales hasta ahora destacadas conforman la matriz cultural desde donde Segundo piensa y ofrece para ser vivido el mensaje salvífico de Jesús. Pero, en definitiva, ¿cómo y con qué signos se entiende la salvación misma?

\section{ACCIÓN HISTÓRICA EN COMÚN VS. OBJETIVO INDIVIDUAL META- HISTÓRICO}

En la obra teológica de Segundo, la salvación se presenta, en primer lugar y sobre todo, como una "performancia", de acuerdo con el lenguaje semiótico utilizado. En efecto, los significantes o signos que se utilizan para referirse a la salvación son especialmente verbos que recubren unas acciones (inclinarse sobre el que sufre, liberar, crear proyectos de amor, etc.) que tienen lugar en la historia. Se trata de acciones que unos seres humanos realizan o han de realizar en favor de otros, de aquellos que son víctimas de los males puestos de relieve; de acciones que conducen el universo dejado a medias por Dios, y entregado a los hombres, hacia su plenitud. Al llevar a cabo esas acciones, quienes lo hacen "colaboran" con Dios y su plan, sean o no conscientes de ello. De ahí que puedan ser cristianos o no cristianos los sujetos de tales acciones. Por esta relación de colaboración, cuando hay hombres que salvan a otros, es Dios mismo quien también salva: la acción de esos hombres se convierte, entonces, en signo del "amor compasivo y activo de Dios".

Esta concepción de la salvación aparece en pugna con otra que sería mayoritaria en América Latina y que entiende la salvación como algo que se ha de conseguir privada o individualmente en la meta-historia, ignorando lo que pasa en la historia. 
Para hacer frente a esa mentalidad y suscitar un cambio cultural, Segundo realiza un proceso de "negociación" del significado de salvación presentando, por ejemplo, a Jesús como modelo -"código" o norma, en lenguaje semiótico- para la acción salvífica en el escenario latinoamericano: Jesús, en su escenario, realiza acciones a favor de otros (sana, libera, acoge, discute...), cumpliendo el programa o plan de Dios hasta el final, hasta la muerte.

Las acciones del o los protagonistas suelen ser evaluadas en lo que en análisis semiótico denomina la "fase de sanción" o retribución de un relato o texto (20). Interesa destacar aquí que, en el discurso teológico de Segundo, lo acontecido con Jesús en la fase de "sanción" también funciona como "código" o norma para evaluar las acciones en otros escenarios. Brevemente, esta fase de sanción se presenta de la siguiente manera: así como a Jesús se le experimentó retribuido ("resucitado") por Dios y, a lo pretendido con su acción histórica, revestido con el valor de lo "definitivo", así también sucederá con los sujetos y acciones de otras historias. El desenlace de la historia de Jesús anticipa, no visible pero sí experiencialmente, lo que acontecerá en las demás historias...

Por otra parte, si bien el signo "salvación" y equivalentes (v. g., "liberación") se asocia preferentemente con una performancia o acciones -liberar, crear proyectos de amor, etc.-, hay que decir que en el corpus estudiado también se utiliza dicho signo para recubrir el "destino" ultraterreno y ultrahistórico del hombre, de todos los hombres. También aquí se marca la diferencia con el discurso predominante en América Latina: en Segundo la vida y felicidad ultraterrena se presenta como un don gratuito de Dios, como una "promesa" en la que hay que creer y no buscar privada o individualmente, de espaldas al plan de Dios ("reino" en términos de Jesús, "construcción" o "agricultura" en términos de Pablo) y a la "situación terriblemente inhumana de sus habitantes". La salvación, tal como aquí se propone, incluye no solo el "destino" eterno de todos los hombres; implica también la "manifestación" de los proyectos "de amor y solidaridad" creados en la historia por los hombres y que parecían fracasar en ella. Dios, al igual que aconteció con Jesús, revestirá con el valor de lo "definitivo" todos esos esfuerzos que pretenden, ya sea liberar a los hermanos necesitados, ya sea crear lo que Dios ha dejado incompleto en el universo. Es decir, en lenguaje semiótico, la salvación corresponde también a la "fase de sanción" puesta de relieve más arriba.

En suma: el significado de la salvación propuesto en este discurso teológico remite a un tipo de acción humana realizada en y de cara a la historia y, también, a la evaluación que se hará de dicha acción. Esta evaluación, en los textos, se desdobla en dos etapas: de manera "imperfecta" se da en el espacio y tiempo y, de manera "perfecta", más allá del espacio y el tiempo: en el "cielo nuevo y la tierra nueva" donde Dios y cada ser humano aportará lo suyo.

Por último, digamos que el signo que se privilegia en los textos para significar la acción y el destino de la acción, es "liberación". Hemos dicho al comienzo que Segundo forma parte del colectivo de los teólogos de la liberación; colectivo que, desde los años sesenta en adelante, ha venido negociando la forma de entender la

(20) Una versión sencilla del método utilizado se puede ver en: GRUPO DE ENTREVERNES, Análisis semiótico de los textos. Introducción, teoría y práctica, Ed. Cristiandad, Madrid 1982. 
salvación en América Latina. Para dar cuenta de la experiencia salvífica de Dios que se tiene en el continente, dicho colectivo ha privilegiado el término "liberación". Desde el punto de vista del análisis cultural, el signo "liberación" reúne las características de lo que en análisis semiótico se denomina "metáfora radical": un signo que, tomado de un determinado campo semántico, empieza también a gobernar otros campos semánticos. Mediante el énfasis en el signo o término "liberación", la experiencia de Dios generadora de nuevas posibilidades para el hombre ha echado raíces en un campo que importa sobremanera en América Latina y que predomina sobre otros: el campo de las relaciones socioeconómicas, allí donde estarían la experiencia de mayor negatividad en el continente. Mediante este procedimiento simbólico, la experiencia salvífica de Dios pasa a ser culturalmente relevante: ella tiene que ver con lo que está en juego en América Latina; ella queda expresada en un lenguaje que todos entienden... Algo parecido aconteció con las comunidades cristianas primitivas las que, en contexto esclavista, se sirvieron y enfatizaron el signo "redención" para dar cuenta de lo bueno acontecido con Jesucristo.

\section{REFLEXIONES FINALES}

Las mediaciones culturales para significar la salvación cristiana desentrañadas en la obra de Juan Luis Segundo, nos hacen caer en la cuenta de que este notable teólogo latinoamericano intentó pensar y expresar la fe atendiendo a los paradigmas vigentes en las sociedades marcadas por la Modernidad. Mediante su teología pretendió liberar la fe cristiana de aquellos paradigmas añejos e incluso contraproducentes, para situarla en nuevas coordenadas socioculturales, de modo que se manifestara todo su potencial y pertinencia. Intentó que la fe cristiana fuera creíble y operativa; que estuviera, en definitiva, "inculturada" en la mentalidad moderna. Ante la vertiginosidad de los cambios culturales que estamos viviendo, es posible que nos parezca que los paradigmas utilizados por Segundo son insuficientes, que sobran unos y que faltan otros. Sin embargo, su esfuerzo por acortar la brecha entre Evangelio y cultura merece ser recogido por la Iglesia Latinoamericana que se reunirá en Brasil para discernir cómo se ha de vivir, anunciar y celebrar la fe en Jesucristo, ante a los nuevos paradigmas culturales que se van instalando en nuestras sociedades globalizadas.

He señalado más arriba que, siendo Juan Luis Segundo un cofundador y genuino representante de la corriente llamada teología de la liberación, en su momento marcó ciertas diferencias con algunas corrientes. Intentando comprender, desde la perspectiva semiótica, la principal diferencia entre el discurso de Segundo con el de otros representantes de la teología de la liberación (en particular, con el de Gustavo Gutiérrez), diría que se encuentra en el papel atribuido a los "pobres" ("beneficiarios" en Segundo; "protagonista" y "beneficiarios" para Gutiérrez). A mi juicio, esa diferencia estaría condicionada por los interlocutores de cada uno de estos teólogos profesionales, por las comunidades eclesiales a las que ellos acompañan. Para Segundo, sus interlocutores fueron comunidades cristianas de clase media urbana. En Teología abierta, por ejemplo, los caracteriza como "franja de la población latinoamericana que era culturalmente europea, pero también terriblemente cuestionada por la situación inhumana de la que junto a ella, no lo era". La califica también de "universitarios, 
jóvenes profesionales, movimientos especializados de la Acción Católica”. Con ellos, añade Segundo, "se vivieron los comienzos de la después llamada "teología de la liberación"” (21). En cambio, para Gustavo Gutiérrez sus principales interlocutores serían comunidades cristianas constituidas por gente del pueblo pobre (22). En la tarea de acompañar a estas comunidades de distinta condición social, ambos teólogos han pretendido ayudar a sus miembros a configurar su identidad y a enfrentar los cambios que todos (clase media y pueblo pobre) parecen percibir como necesarios, a la luz de la palabra de Dios. Y, al hacerlo, da la impresión de que no pueden sino situar a sus interlocutores en la posición de "protagonista". Porque, en definitiva, cuando una teología quiere ser ayuda para que se consolide la identidad (cultural y cristiana) de un colectivo humano y para que se enfrenten los cambios percibidos como necesarios, la "dignidad" que está en juego para ese colectivo implica que se les sitúe como sujetos de su propia historia. Hubo, pues, un reclamo de pluralismo dentro de la teología de la liberación, debido a la diversidad de colectivos humanos (y sus respectivas culturas) en la que dicha teología se fraguó. Pluralismo al que en su momento no se supo responder. Pluralismo que, en una sociedad globalizada como la actual, urge reconocer e incluso promover para el enriquecimiento de todos en la Iglesia.

\section{RESUMEN}

Como una contribución a la V Conferencia General del Episcopado Latinoamericano y del Caribe, el artículo retoma el legado teológico del Juan Luis Segundo (1925-1996). Este notable teólogo uruguayo contribuyó, como pocos, a acortar la brecha o "ruptura entre Evangelio y cultura" que tanto preocupaba a Pablo VI. Ruptura que pareciera haberse ahondado nuevamente en nuestros días. En el marco de las anteriores Conferencias Generales del CELAM, Segundo no solo urgió a liberar la teología y la pastoral de paradigmas obsoletos y contraproducentes sino que, además, contribuyó a expresar el Evangelio en nuevas coordenadas culturales. En particular, en paradigmas más cercanos a la cultura moderna que marcaban a amplios sectores, sobre todo urbanos, de la región. Recoger el legado teológico de Segundo es una forma también de recuperar el quehacer teológico como mediación indispensable para llevar a cabo la urgente tarea de articular Evangelio y culturas.

\section{ABSTRACT}

As a contribution to the Fifth General Conference of Latin-American and Caribbean Bishops, this article proposes to recover the theological legacy of Juan Luis Segundo (19251996). This Uruguayan theologian helped, as few were able to do, to shrink the gap, or "rupture", between Gospel and culture that so preoccupied Paul VI, a rupture that nowadays seems to have deepened once again. Within the framework of the previous General Conferences, Segundo not only urged the liberation of theology and pastoral practice from aged and counterproductive paradigms, but also helped to express the Gospel within new cultural frameworks-in particular, in paradigms closer to the modern culture that shaped wide sectors (mostly urban) of the region. To recover Segundo's theological legacy is also a way to recover theology as an indispensable mediation for accomplishing the urgent task of articulating Gospel and cultures.

(21) Ob. cit., tomo III, pp. 16-17.

(22) Véase, por ejemplo, "Mirar lejos", que es la introducción a la edición revisada y aumentada de Teología de la liberación. Perspectivas, Ed. Sígueme, Salamanca ${ }^{14} 1990$. 